\title{
72.
}

\section{NOTE ON THE THEORY OF PERMUTATIONS.}

[From the Philosophical Magazine, vol. xxxiv. (1849), pp. 527-529.]

IT seems worth inquiring whether the distinction made use of in the theory of determinants, of the permutations of a series of things all of them different, into positive and negative permutations, can be made in the case of a series of things not all of them different. The ordinary rule is well known, viz. permutations are considered as positive or negative according as they are derived from the primitive arrangement by an even or an odd number of inversions (that is, interchanges of two things); and it is obvious that this rule fails when two or more of the series of things become identical, since in this case any given permutation can be derived indifferently by means of an even or an odd number of inversions. To state the rule in a different form, it will be convenient to enter into some preliminary explanations. Consider a series of $n$ things, all of them different, and let $a b c \ldots$ be the primitive arrangement; imagine a symbol such as $(x y z)(u)(v w) \ldots$ where $x, y$, \&c., are the entire series of $n$ things, and which symbol is to be considered as furnishing a rule by which a permutation is to be derived from the primitive arrangement $a b c \ldots$ as follows, viz. the $(x y z)$ of the symbol denotes that the letters $x, y, z$ in the primitive arrangement $a b c \ldots$ are to be interchanged $x$ into $y, y$ into $z, z$ into $x$. The $(u)$ of the symbol denotes that the letter $u$ in the primitive arrangement $a b c \ldots$ is to remain unaltered. The $(v w)$ of the symbol denotes that the letters $v, w$ in the primitive arrangement are to be interchanged $v$ into $w$ and $w$ into $v$, and so on. It is easily seen that any permutation whatever can be derived (and derived in one manner only) from the primitive arrangement by means of a rule such as is furnished by the symbol in question ${ }^{1}$; and moreover that the number of inversions requisite in order to obtain the permutation by means of the rule in question, is always the smallest number of

${ }^{1}$ See on this subject Cauchy's "Mémoire sur les Arrangemens \&c.", Exercises d'Analyse et de Physique Mathématique, t. III. [1844], p. 151. 
inversions by which the permutation can be derived. Let $\alpha, \beta \ldots$ be the number of letters in the components $(x y z),(u)(v w), \& c ., \lambda$ the number of these components. The number of inversions in question is evidently $\overline{\alpha-1}+\overline{\beta-1}+\&$ c., or what comes to the same thing, this number is $(n-\lambda)$. It will be convenient to term this number $\lambda$ the exponent of irregularity of the permutation, and then $(n-\lambda)$ may be termed the supplement of the exponent of irregularity. The rule in the case of a series of things, all of them different, may consequently be stated as follows: "a permutation is positive or negative according as the supplement of the exponent of irregularity is even or odd." Consider now a series of things, not all of them different, and suppose that this is derived from the system of the same number of things $a b c \ldots$ all of them originally different, by supposing for instance $a=b=\& c$., $f=g=\&$ c. A given permutation of the system of things not all of them different, is of course derivable under the supposition in question from several different permutations of the series $a b c \ldots$. Considering the supplements of the exponents of irregularity of these last-mentioned several permutations, we may consider the given permutation as positive or negative according as the least of these numbers is even or odd. Hence we obtain the rule, "a permutation of a series of things not all of them different, is positive or negative according as the minimum supplement of irregularity of the permutation is even or odd, the system being considered as a particular case of a system of the same number of things all of them different, and the given permutation being successively considered as derived from the different permutations which upon this supposition reduce themselves to the given permutation." This only differs from the rule, "a permutation of a series of things, not all of them different, is positive or negative according as the minimum number of inversions by which it can be obtained is even or odd, the system being considered \&c.," inasmuch as the former enunciation is based upon and indicates a direct method of determining the minimum number of inversions requisite in order to obtain a given permutation; but the latter is, in simple cases, of the easier application. As a very simple example, treated by the former rule, we may consider the permutation 1212 derived from the primitive arrangement 1122. Considering this primitive arrangement as a particular case of $a b c d$, there are four permutations which, on the suppositions $a=b=1, c=d=2$, reduce themselves to 1212 , viz. $a c b d$, $b c a d, a d b c$, $b d a c$, which are obtained by means of the respective symbols $(a)(b c)(d) ;(a b c)(d)$; $(a)(b d c) ;(a b d c)$, the supplements of the exponents of irregularity being therefore $1,2,2,3$, or the permutation being negative; in fact it is obviously derivable by means of an inversion of the two mean terms. 